\title{
Um método iterativo eficiente para resolver sistemas de equações pentadiagonais
}

\author{
Diego Fernando Moro ${ }^{1}$ \\ Programa de Pós-Graduação em Engenharia Mecânica (PGMec) - UFPR \\ Centro Politécnico - UFPR, Curitiba, Paraná, Brasil
}

Carlos Henrique Marchi ${ }^{2}$

Márcio Augusto Villela Pinto

Departamento de Engenharia Mecânica - UFPR

Centro Politécnico - UFPR, Curitiba, Paraná, Brasil

\begin{abstract}
Resumo. É proposto e testado um algoritmo para resolver sistemas de equações pentadiagonais esparsos. Ele é baseado no TDMA (Tridiagonal Matrix Algorithm), sendo denominado neste trabalho de PDMA (Pentadiagonal Matrix Algorithm). Este sistema de equações possui duas diagonais lado a lado da diagonal principal da matriz e mais duas diagonais não nulas. A dedução mostrada neste trabalho pode ser feita para qualquer número de diagonais na matriz de coeficientes. O método PDMA foi testado na solução das equações de Laplace 2D e Burgers 2D. Os resultados foram comparados com outros métodos da literatura: Gauss-Seidel, ADI (Alternating Direction Implicit), TDMAX (a direção axial é explicita e a direção transversal é implícita) e MSI (Modified Strongly Implicit). Para as equações de Burgers, em uma malha de 256x256 volumes, o PDMA resolveu o problema em menos de $11 \%$ do tempo do método MSI, que por sua vez resolveu o problema em cerca de $12 \%$ do ADI.

Palavras-chave. Métodos iterativos, transferência de calor computacional, dinâmica dos fluidos computacional, sistema de equações lineares, solver
\end{abstract}

\section{Introdução}

A motivação para este trabalho foi a constante busca para diminuir o tempo computacional necessário para obter soluções numéricas.

Ao observar as equações do método iterativo (solver) ADI (Alternating Direction Implicit) [3], que utilizam uma dedução do TDMA (Tridiagonal Matrix Algorithm) [7] implícito em uma direção do plano e explícito na outra direção e vice-versa, surgiu a ideia de acoplar essas duas deduções em uma única fórmula e deduzir coeficientes para resolver o sistema linear pentadiagonal.

\footnotetext{
${ }^{1}$ difmoro@ufpr.br, difmoro@gmail.com

2 marchi@ufpr.br,chmcfd@gmail.com

3 marcio_villela@ufpr.br
} 


\section{Metodologia}

O objetivo do solver PDMA (Pentadiagonal Matrix Algorithm), proposto neste trabalho, é a resolução de sistemas de equações cuja matriz de coeficientes seja do tipo pentadiagonal, ou seja, possui apenas 5 diagonais não nulas na matriz dos coeficientes. A matriz mencionada pode ser originada, por exemplo, da discretização via volumes finitos de uma malha bidimensional onde 2 diagonais são distantes da diagonal principal com nx coeficientes de distância, sendo que nx é o número de volumes na direção axial.

A equação geral deste tipo de sistema de equações é dado pela expressão:

$$
a_{p} X_{p}+a_{e} X_{E}+a_{w} X_{W}+a_{n} X_{N}+a_{s} X_{S}=b_{p} .
$$

Sendo que:

$X_{p, E, W, N, S}$ : Incógnita, com a indicação da posição em relação a diagonal principal.

$a_{p, e, w, n, s}:$ Coeficientes do sistema de equações, com a indicação da posição do coeficiente em relação à diagonal principal.

$b_{p}$ : Termo fonte do sistema linear.

\subsection{Equações genéricas para leste e norte}

Neste trabalho será introduzido o método PDMA, utilizando-se duas equações genéricas com as seguintes expressões:

$$
\begin{aligned}
& X_{p}=V_{1, p} X_{E}+V_{4, p} . \\
& X_{p}=V_{2, p} X_{N}+V_{5, p} .
\end{aligned}
$$

Sendo que:

$V_{i, p}, \mathrm{i}=1,2$, 4 e 5: Vetores incógnita do método PDMA, $p$ é o índice em relação aos vetores incógnita do método PDMA.

O método PDMA consiste em deixar a Eq. (1) dependendo apenas das duas direções do plano, tanto Leste (E) quanto Norte (N). Basta portanto deslocar as Eqs. (2) e (3) para Oeste (W) e Sul (S), respectivamente. Teremos:

$$
\begin{gathered}
X_{W}=V_{1, W} X_{p}+V_{4, W} . \\
X_{S}=V_{2, S} X_{p}+V_{5, S}
\end{gathered}
$$

Substituindo-se as Eqs. (4) e (5) na Eq. (1) e isolando os coeficientes que multiplicam as incógnitas $\mathrm{X}_{\mathrm{E}}$ e $\mathrm{X}_{\mathrm{N}}$ podemos encontrar os valores dos vetores incógnita do método PDMA. A equação fica da forma 


$$
X_{p}=\frac{V_{1, p}}{2} X_{e}+\frac{V_{2, p}}{2} X_{n}+\frac{V_{3, p}}{2}
$$

Sendo que $\mathrm{V}_{3, p}$ é a soma dos vetores $\mathrm{V}_{4, p}$ e $\mathrm{V}_{5, p}$, os vetores incógnitas $\mathrm{V}_{1, p}$ e $\mathrm{V}_{2, p}$ ficam na forma das Eqs. (7) a (9).

\subsection{Equações para os vetores incógnitas}

$$
\begin{gathered}
V_{1, p}=\frac{-2 a_{e}}{a_{p}+a_{w} V_{1, w}+a_{s} V_{2, s}} \\
V_{2, p}=\frac{-2 a_{n}}{a_{p}+a_{w} V_{1, w}+a_{s} V_{2, s}} \\
V_{3, p}=2 \frac{b_{p}-a_{w} V_{4, w}-a_{s} V_{5, s}}{a_{p}+a_{w} V_{1, w}+a_{s} V_{2, s}}
\end{gathered}
$$

$\mathrm{Na}$ Eq. (9), os vetores $\mathrm{V}_{4, W}$ e $\mathrm{V}_{5, S}$ não são conhecidos, mas se os isolarmos nas Eqs. (4) e (5) e substituirmos, podemos encontrar uma expressão para $V_{3, p}$ que depende apenas de vetores calculados, desta forma teremos a Eq. (10):

$$
V_{3, p}=2 \frac{b_{p}-a_{w}\left(X_{w}-V_{1, w} X_{p}\right)-a_{s}\left(X_{s}-V_{2, s} X_{p}\right)}{a_{p}+a_{w} V_{1, w}+a_{s} V_{2, s}}
$$

Agora é possível calcular os vetores incógnitas do método PDMA seguindo a ordenação lexicográfica. Como é possível observar, o terceiro vetor incógnita do método PDMA, Eq. (10), depende da solução, nos pontos W, S e no ponto P, ou seja, o solver se torna iterativo.

\subsection{Algoritmo}

O algoritmo parte do princípio que a matriz utilizada foi obtida utilizando-se a ordem lexicográfica.

1) Estima-se um campo de valores para a variável dependente incógnita;

2) Calcula-se os vetores incógnitas do PDMA com as Eqs. (7), (8), e (10) notando os casos especiais, onde $\mathrm{V}_{1, w}, \mathrm{~V}_{2, s}, \mathrm{X}_{w}, \mathrm{X}_{s}$ não existem, calculando-se estas incógnitas em ciclo progressivo na matriz dos coeficientes;

3) Em ciclo regressivo, resolve-se o sistema linear para a variável dependente com a Eq. (6);

4) Volta-se ao passo 2 até atingir uma tolerância. 


\section{Modelos matemáticos e numéricos}

\subsection{Equações de Laplace e Burgers}

Para as simplificações: condução de calor bidimensional, regime permanente, ausência de geração de calor, teremos a equação de Laplace, Eq. (11), que é a equação diferencial que modela o problema. O domínio é uma placa quadrada unitária e as condições de contorno estão na Eq. (12)

$$
\begin{gathered}
\frac{\partial^{2} T}{\partial x^{2}}+\frac{\partial^{2} T}{\partial y^{2}}=0 \\
\left\{\begin{array}{c}
T(0, y)=T(1, y)=T(x, 0)=0 \\
T(x, 1)=\operatorname{sen}(\pi x)
\end{array}\right.
\end{gathered}
$$

Considerando um escoamento bidimensional de fluido incompressível com propriedades constantes, as equações diferenciais que modelam o problema são as equações de Burgers, Eq. (13). O domínio também é uma placa quadrada unitária e as condições de contorno estão na Eq. (14):

$$
\left\{\begin{array}{c}
\rho \frac{\partial\left(u^{2}\right)}{\partial x}+\rho \frac{\partial(u v)}{\partial y}=\mu\left(\frac{\partial^{2} u}{\partial x^{2}}+\frac{\partial^{2} u}{\partial y^{2}}\right)-\frac{\partial p}{\partial x} \\
\rho \frac{\partial(u v)}{\partial x}+\rho \frac{\partial\left(v^{2}\right)}{\partial y}=\mu\left(\frac{\partial^{2} v}{\partial x^{2}}+\frac{\partial^{2} v}{\partial y^{2}}\right)-\frac{\partial p}{\partial y}-B(x, y, \operatorname{Re}=1) \\
\left\{\begin{array}{l}
u(0, y)=u(1, y)=u(x, 0)=0 \\
u(x, 1)=16\left(x^{4}-2 x^{3}+x^{2}\right) \\
v(0, y)=v(1, y)=v(x, 0)=v(x, 1)=0
\end{array}\right.
\end{array}\right.
$$

$\mathrm{O}$ termo $\mathrm{B}(x, y, \mathrm{Re}=1)$ na Eq. (13) foi utilizado para encontrar uma solução analítica (neste caso, utilizou-se o método das soluções fabricadas), juntamente com o campo de pressão obtidos no artigo de Shih et al [5].

Dados numéricos para as duas equações:

- O método numérico utilizado para discretizar a equação diferencial é o método dos volumes finitos (MVF).

- As aproximações utilizadas para as derivadas são de segunda ordem de acurácia (Central Difference Scheme, CDS).

- As condições de contorno são aplicadas utilizando-se volumes fictícios, com o valor no contorno conhecido [1].

- A estimativa inicial do campo de temperaturas ou velocidades é nulo.

- O critério de parada do método iterativo na resolução do sistema linear, se dá com base numa tolerância sobre o resíduo adimensionalizado (razão entre o resíduo atual 
pelo resíduo da primeira iteração) do sistema linear e a tolerância utilizada é de $1.10^{-10}$.

\subsection{Solvers e método de comparação dos resultados}

Para a comparação do solver deste trabalho com outros da literatura, selecionou-se os seguintes: (1) Gauss-Seidel, (2) Alternating Direction Implicit (ADI) [3], (3) TDMA com a Direção Y Implícita e (4) Modified Strongly Implicit (MSI) [4].

Para cada tamanho de problema n é possível encontrar a expressão que representa o comportamento do tempo de CPU utilizado no solver da seguinte forma:

$$
t_{C P U}=a n^{p}
$$

Sendo que geralmente as constantes $a$ e $p$ representam o desempenho de um determinado solver, aliado às características da programação utilizada e $n$ é o número de incógnitas do sistema de equações. Sabe-se experimentalmente que os solvers utilizados para a comparação do PDMA neste trabalho possuem o expoente $p$ da Eq. (15) próximo a 2. Segundo [6], o método Gauss-Seidel com multigrid resulta em um expoente $p$ próximo a 1 .

\subsection{Dedução do PDMA para outros pares de volumes}

Ao considerar outros pares de volumes nas Eqs. (2) e (3), por exemplo $\mathrm{N}$ e W, W e S e $\mathrm{S}$ e $\mathrm{E}$ e aplicar o mesmo procedimento mostrado, obteremos outros 3 solvers distintos, que podem influenciar no tempo de CPU se utilizados em conjunto.

Neste trabalho as deduções do método PDMA são chamadas: PDMA-EN (dedução demostrada neste trabalho com E e N), PDMA-EN-NW (uso do solver PDMA-EN com a dedução N e W utilizada em seguida), PDMA-EN-NS-WS (uso do solver PDMA-EN, PDMA-NS e do PDMA-WS um em seguida do outro nesta ordem).

\section{Resultados e conclusão}

\subsection{Equação de Laplace e Burgers}

A comparação dos resultados dos solvers da literatura e o PDMA estão nas Figs.1 e 2 e na Tab. 1.

Neste trabalho foi proposto um novo solver utilizando a média de duas deduções do método TDMA. Foi estudado também o desempenho deste solver frente a outros solvers da literatura em dois problemas: equação de Laplace e equações de Burgers. Os solvers da literatura utilizados foram: Gauss-Seidel, ADI, TDMAX, MSI. Foi realizado também uma análise quanto ao uso conjunto de 2 e 3 deduções do PDMA. 
Tabela 1: Constantes da Eq. (15) para as equações de Laplace e Burgers.

\begin{tabular}{|c|c|c|}
\hline Laplace & $\boldsymbol{p}$ & $\boldsymbol{a}$ \\
\hline PDMA-EN & 1.563 & $4.190 \mathrm{E}-08$ \\
\hline PDMA-EN-NW & 1.496 & $7.184 \mathrm{E}-08$ \\
\hline PDMA-EN-NW-WS-SE & 1.521 & $5.646 \mathrm{E}-08$ \\
\hline GS & 1.988 & $1.840 \mathrm{E}-08$ \\
\hline ADI & 1.991 & $1.727 \mathrm{E}-08$ \\
\hline TDMAX & 1.934 & $2.322 \mathrm{E}-08$ \\
\hline MSI & 1.934 & $5.573 \mathrm{E}-09$ \\
\hline
\end{tabular}

\begin{tabular}{|c|c|c|}
\hline Burgers & $\boldsymbol{p}$ & $\boldsymbol{a}$ \\
\hline PDMA-EN & 1.572 & $8.296 \mathrm{E}-08$ \\
\hline PDMA-EN-NW & 1.515 & $1.163 \mathrm{E}-07$ \\
\hline PDMA-EN-NW-WS-SE & 1.557 & $8.764 \mathrm{E}-08$ \\
\hline GS & 2.011 & $4.393 \mathrm{E}-08$ \\
\hline ADI & 2.001 & $3.181 \mathrm{E}-08$ \\
\hline TDMAX & 1.979 & $4.409 \mathrm{E}-08$ \\
\hline MSI & 1.828 & $2.444 \mathrm{E}-08$ \\
\hline
\end{tabular}
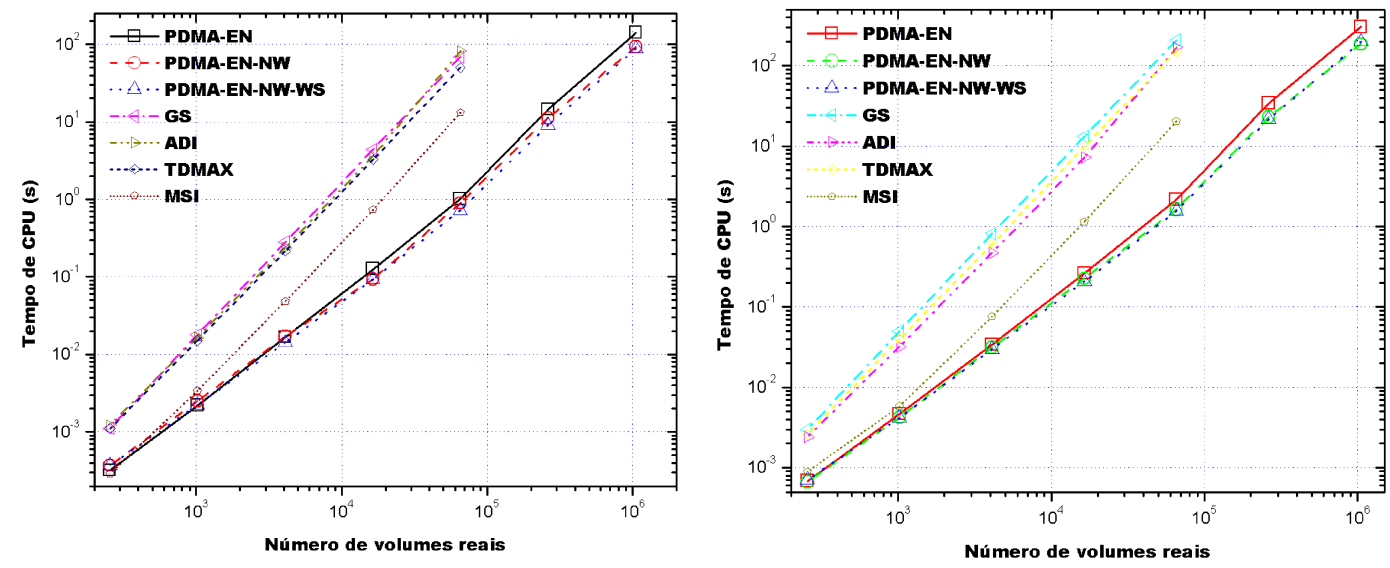

Figura 1: Comparação entre os tempos de CPU para as equações de Laplace (esquerda) e de Burgers (direita).

Com base nos testes realizados, pode-se concluir que:

1 - Todas as deduções do PDMA propostas, convergiram mais rápido do que os solvers da literatura utilizados neste trabalho.

Na malha 256x256 das equações de Burgers, o método PDMA-EN convergiu em $10,69 \%$ do tempo necessário para o MSI (melhor solver da literatura utilizado), que por sua vez convergiu em $11,94 \%$ do tempo necessário para o solver ADI.

2 - Utilizando-se o PDMA em conjunto, o tempo computacional diminui consideravelmente: na malha mais fina simulada para as equações de Burgers, o solver PDMA-EN-NW-WS resolveu o problema $34,32 \%$ mais rápido que o solver PDMA-EN. O solver PDMA-EN-NW foi 37,95\% mais rápido que o PDMA-EN.

3 - $\mathrm{O}$ uso do solver proposto neste trabalho é mais rápido que os solvers da literatura utilizados.

4 - Com base na Eq. (15), o expoente $p$ para todas as versões do PDMA aproxima-se de 1,5 .

Este valor do expoente $\mathrm{p}$ torna o solver deste trabalho mais próximo do solver Gauss-Seidel com multigrid (no qual o expoente desta curva se aproxima de 1,0) e 
melhor do que todos os outros solvers da literatura testados neste trabalho (que se aproximam de 2,0).

Lembrando que para um mesmo expoente, a diferença de tempo computacional se encontra no número de operações necessárias para o determinado solver. No entanto a mudança neste expoente se reflete em um comportamento diferente do tempo de CPU, tornando o solver mais rápido que os outros com o refino da malha.

Foi constatado que para problemas com condições de contorno diferentes de Dirichlet (valor conhecido no contorno) o solver não funciona, gerando divisão por zero em alguns pontos da malha. É possível, no entanto, utilizá-lo apenas para o interior do domínio e os contornos resolvidos com Gauss-Seidel, desta forma o solver funciona mas é mais parecido com os solvers tradicionais da literatura.

\section{Agradecimentos}

Os autores agradecem o apoio financeiro do CNPq (Conselho Nacional de Desenvolvimento Científico e Tecnológico) e CAPES (Coordenação de Aperfeiçoamento de Pessoal de Nível Superior). O primeiro autor é bolsista da CAPES. O segundo autor é bolsista do CNPq.

\section{Referências}

[1] S. C Chapra e R. P. Canale, Métodos Numéricos para Engenharia, 5 Ed., McGrawHill, São Paulo (2008).

[2] F. P. Incropera e D. P. DeWitt, Introduction to Heat Transfer, 3rd Ed., Wiley, New York (1996).

[3] D. W. Peaceman and H. H. Rachford, The Numerical Solution of Parabolic and Eliptic Differential Equations, J. Soc. Ind. Appl. Math, vol. 3, pp. 28-41 (1955).

[4] G. Schneider and M. Zedan, A modified strongly implicit procedure for the numerical solution of field problems. Numerical Heat Transfer, vol. 4, pp. 1-19 (1981).

[5] T. M. Shih, C. H. Tan and B. C. Hwang, Effects of Grid Staggering on Numerical Schemes, Int. J. Numer. Meth. Fluids, vol. 9, pp. 193-212 (1989).

[6] R. Suero, M. A. V. Pinto, C. H. Marchi, L. K. Araki e A. C. Alves, Otimização do método multigrid algébrico para as equações bidimensionais de Laplace e Poisson, VI Congresso Nacional de Engenharia Mecânica, Paraíba, Brasil (2010).

[7] H. K. Versteeg and W. Malalasekera, An Introduction to Computational Fluid Dynamics - The Finite Volume Method, Pearson, Prentice Hall, 2. Ed, (2007). 